\title{
Der Anstieg des Tritiumgehaltes im atmosphärischen Wasserstoff seit 1960
}

\author{
D. EhHaLt *, W. Roether und W. Stich ** \\ II. Physikalisches Institut der Universität Heidelberg, C-14 Laboratorium \\ (Z. Naturforschg. 21 a, 1703-1709 [1966] ; eingegangen am 3. Juni 1966)
}

\begin{abstract}
Measurements of the tritium and deuterium content of atmospheric hydrogen are reported. The measured tritium is corrected for local contamination by industrial hydrogen. The samples cover the period from 1960 to 1965 . The tritium content shows a marked increase in 1961 but rises only slowly during 1962 and 1963 . There is evidence for a further increase in the tritium content starting in fall 1964. The explanation suggested is, that tritiated hydrogen is injected into the high stratosphere by thermonuclear explosions and mixed down into the troposphere with a $2-3$ years delay. Thus one would ascribe the first increase to 1958 testing, the second to the $1961 / 1962$ thermonuclear test series.
\end{abstract}

Seit den ersten thermonuklearen Explosionen (Ivy Test 1952) ist die Tritium-Aktivität im atmosphärischen Wasserstoff mehr oder weniger kontinuierlich angewachsen. Sie beträgt inzwischen mit etwa $5 \cdot 10^{6} \mathrm{TU}^{1}$ etwa das Tausendfache des natürlichen Pegels vor 1952. Ob diese künstliche T-Aktivität durch die nuklearen Testexplosionen injiziert oder direkt durch die anwachsende Kernindustrie abgegeben wurde, ist noch nicht eindeutig geklärt. Für Tritium im atmosphärischen Wasserstoff wurde bislang keine direkte Korrelation zwischen Testintensität und nachfolgendem Aktivitätsanstieg gefunden, wie sie bei allen übrigen bombenerzeugten Nukliden vorliegt. Die Spaltprodukte, Kohlenstoff-14 in atmosphärischem $\mathrm{CO}_{2}$, aber auch Tritium im Wasserdampf (Regen) zeigen ausnahmslos einen Aktivitätsanstieg in dem auf den Test folgenden Jahr. Dieses Verhalten ist auf die Injektion von radioaktivem Material in die untere Stratosphäre zurückzuführen, von wo es bereits im folgenden Frühjahr in die Troposphäre heruntergemischt wird. Das Ausbleiben eines solchen rasch nachfolgenden Aktivitätsanstiegs für Tritium im Wasserstoff, ferner die Ähnlichkeit des Tritium-Anstieges mit dem von Kr-85, das hauptsächlich durch die Kernindustrie abgegeben wird ${ }^{2,3}$, schienen darauf hinzuweisen, daß auch ein beträchtlicher Anteil des Tritium im Wasserstoff kernindustrieller Herkunft sei ${ }^{4}$. Demgegenüber zeigt die Diskussion der im folgenden beschriebenen Messun-

* Zur Zeit am National Center for Atmospheric Research, Boulder, Colorado.

** Linde Aktiengesellschaft, Werksgruppe München-Lohhof, Bayern.

11 TU (Tritium unit) bedeutet eine T-Konzentration von 1 T-Atom pro $10^{18}$ Atome Wasserstoff.

2 D. Ehhalt, K. O. Münnich, W. Rogther, J. Schölch u. W. Stich, J. Geophys. Res. 68, 3817 [1963]. gen die Möglichkeit auf, daß ein wesentlicher Teil des Anstiegs seit 1960 den Testserien 1958 und seit 1964 den Explosionen 1961, 1962 zuzuschreiben ist.

\section{Probengewinnung und Meßmethode}

Bei der Luftverflüssigung wird der atmosphärische Wasserstoff mit den nichtkondensierbaren Edelgasen in der sogenannten Rohneonfraktion angereichert. Die untersuchten Gasproben wurden in den Luftverflüssigungsanlagen von Lohhof (bei München) und Oberhausen entnommen. Aus dem Gasgemisch, bestehend aus $\mathrm{H}_{2}$ (ca. $1 \%$ ), $\mathrm{He}, \mathrm{Ne}$ und $\mathrm{N}_{2}$, wurde der Wasserstoff anfänglich durch Diffusion durch ein heißes Palladiumrohr abgetrennt. Dazu wurden etwa 71 Gas (500 Torr) in ein zuvor sorgfältig evakuiertes Umwälzsystem eingelassen. Etwaige in dem Gas vorhandene Spuren von Wasser wurden durch eine Kühlfalle beim Einlassen aufgefangen. Anschließend wurde das Gemisch für etwa 6 Stunden durch ein $400{ }^{\circ} \mathrm{C}$ heißes Palladiumrohr umgewälzt. Der durch das Palladiumrohr diffundierte Wasserstoff wurde durch eine Quecksilber-Diffusionspumpe in einen Vorratskolben gepumpt und von dort mittels einer automatischen Tozpler-Pumpe in die Probenampulle gepreßt.

Diese Methode mußte aufgegeben werden, als wir Ende 1963 dazu übergingen, Sammelproben über einen längeren Zeitraum zu entnehmen und es sich herausstellte, daß diese Proben Spuren von Sauerstoff enthielten. Sauerstoff würde mit einem Teil des Wasserstoffs an der Palladium-Oberfläche zu Wasser reagieren und damit eine merkliche Isotopentrennung in dem nicht mehr quantitativ gewonnenen Wasserstoff verursachen.

3 D. Ehhalt, K. O. Münnich, W. Roether, J. Schölch u. W. S тісн, Krypton-85 in the Atmosphere, 3rd Intern. Conf. on the Peaceful Uses of Atomic Energy, Paper A/Conf. 28/P /541, Genf 1964.

4 D. Ehhalt, G. Israel, W. Roether u. W. Stich, J. Geophys. Res. 68, 3747 [1963]. 
Um dieses zu umgehen, wurde das Palladium-Rohr ersetzt durch ein mit $\mathrm{CuO}$ gefülltes Rohr, das den Wasserstoff bei etwa $500{ }^{\circ} \mathrm{C}$ zu Wasser oxydiert. Das Wasser wird durch eine in den Kreislauf geschaltene Kühlfalle aufgefangen. Nach einer Reaktionszeit von etwa 48 Stunden wird das Restgas abgepumpt und das gewonnene Wasser in eine Ampulle übergefroren. Es wird dann nach einer üblichen Methode über heißem Zink von etwa $450{ }^{\circ} \mathrm{C}$ wieder zu Wasserstoff reduziert, der als Probengas sowohl für die Deuterium- als auch für die Tritium-Messung dient ${ }^{5}$. Beide Methoden wurden auf quantitative Ausbeute geprüft, indem der Wasserstoff-Gehalt des Gasgemisches vor und nach der Reaktion massenspektrometrisch untersucht wurde. Ferner wurde die Ausbeute in Abhängigkeit von der Reaktionszeit bestimmt. Die angegebenen Zeiten ergaben eine Ausbeute besser als 99\%. Schließlich wurde die Reproduzierbarkeit geprüft, indem Teile derselben Rohneonprobe nacheinander der Aufbereitung unterzogen wurden. Die D- und T-Gehalte der so gewonnenen Wasserstoff-Proben zeigten gute Übereinstimmung.

Der D-Gehalt des Wasserstoffs wurde in einem kommerziellen Massenspektrometer (Atlas MS 86) bestimmt. Um die erforderliche Genauigkeit zu erhalten, wird nach einer üblichen Methode ${ }^{5}$ der D-Gehalt der Probe mit einem Standard verglichen und dementsprechend als relative Abweichung $\delta$ (in \%00) von diesem Standard angegeben:

$$
\delta=1000 \cdot \frac{R_{\mathrm{P}}-R_{\mathrm{ST}}}{R_{\mathrm{ST}}}, \quad R=\frac{[\mathrm{D}]}{[\mathrm{H}]} .
$$

Nach einem Vorschlag von CraIg (1961) ${ }^{5 a}$ bezieht man die Abweichung auf "standard mean ocean water" (SMOW). Der maximale Fehler beträgt $\Delta \delta= \pm 2 \%$.

Die T-Aktivität wurde in einer speziell für niedere
Aktivitäten eingerichteten Zählapparatur (Proportionalzähler) nachgewiesen. Die Meßmethode sowie apparative Einzelheiten sind bereits beschrieben ${ }^{6}$. Der Meßfehler (Standardabweichung) beträgt im allgemeinen $\pm 5 \%$. Der Fehler stammt hauptsächlich von der Druckablesung, da die Apparatur auf die vorliegenden kleinen Probemengen nicht eingerichtet ist.

Sämtliche Messungen sind in Tab. 1 zusammengestellt.

Die Zusammensetzung der Rohneonproben wurde ebenfalls massenspektrometrisch bestimmt, indem die Proben mit einem Eichgemisch verglichen wurden, dessen Zusammensetzung etwa dem der Proben entsprach. Für das Folgende ist vor allem das $\mathrm{H}_{2} / \mathrm{He}-$ Verhältnis wichtig, das in Tab. 1 ebenfalls aufgeführt ist.

\section{Korrektur der gemessenen T-Werte auf Beimischung von industriellem $\mathrm{H}_{2}$}

Die gemessenen D- und T-Gehalte der in Tab. 1 aufgeführten Proben streuen beträchtlich. Wie BEgEMANN und Friedman ${ }^{7}$ jedoch zeigten, besteht eine lineare Korrelation zwischen dem Gehalt an beiden Isotopen. Diese Korrelation weist auf eine lokale Beimischung von T-freiem Wasserstoff mit niedrigem und annähernd konstantem D-Gehalt hin. Der D-Gehalt des beigemischten Wasserstoffs kann durch Extrapolation der Korrelationsgeraden auf den T-Gehalt Null bestimmt werden. In Abb. 1 ist der T-Gehalt von Proben aus Lohhof gegen den D-Gehalt aufgetragen. Ganz offensichtlich zerfallen die Meßdaten

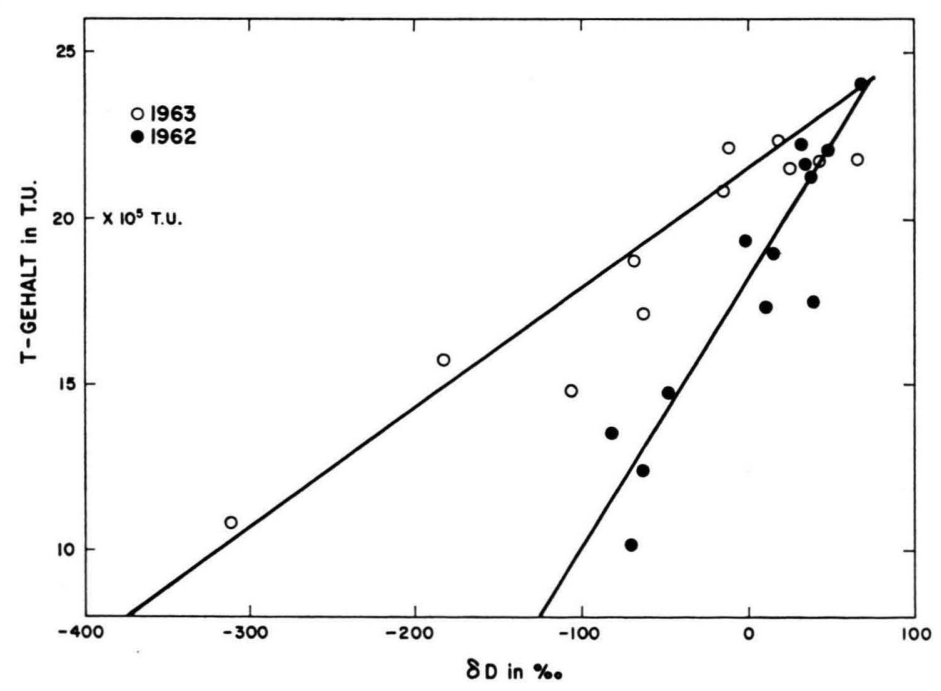

Abb. 1. Korrelation zwischen T- und D-Gehalt. Die Proben wurden während der zweiten Hälfte 1962 (Ne 22-Ne 38) und 1963 entnommen. Offensichtlich folgen die Proben aus 1962 einer anderen Geraden als die 1963 entnommenen (vgl. Text).
5 I. Friedman, Geochim. Cosmochim. Acta 4, 89 [1953].

5 a. Craig, Science 133, 1833 [1961].

${ }^{6}$ D. Ehhalt, K. O. Münnich u. W. Roether, C-14 working paper submitted to the Panel on the "World Wide Survey of Hydrogen and Oxygen Isotopes in Precipitation" held at the IAEA Headquarters in Vienna, 12-16 October 1964.

7 F. Begemann u. I. Friedman, Z. Naturforschg. 14 a, 1024 [1959]. 


\begin{tabular}{|c|c|c|c|c|c|c|c|}
\hline Nr. & Entnahmeort & Uhrzeit & $\begin{array}{c}\text { Entnahme- } \\
\text { datum }\end{array}$ & $\begin{array}{l}\mathrm{H}_{2} / \mathrm{He}- \\
\text { Verhältnis }\end{array}$ & $\begin{array}{c}\text { D-Gehalt } * * \\
\text { in } \% 00\end{array}$ & $\begin{array}{l}\text { T-Gehalt } \\
\text { in } 10^{5} \mathrm{~T} . \mathrm{U} .\end{array}$ & $\begin{array}{c}\text { korrigierter } \\
\text { T-Gehalt } \\
\text { in } 10^{5} \mathrm{~T} . \mathrm{U} .\end{array}$ \\
\hline $\begin{array}{l}\text { L } 101 * \\
\text { L } 102 \\
\text { L } 103 \\
\text { L } 104 \\
\text { L } 105 \\
\text { L } 106 \\
\text { L } 107 \\
\text { L } 108\end{array}$ & $\begin{array}{l}\text { Nürnberg } \\
\text { Witten } \\
\text { Oberhausen } \\
\text { Oberhausen } \\
\text { Oberhausen } \\
\text { Oberhausen } \\
\text { München *** } \\
\text { Mü cchen *** }\end{array}$ & & $\begin{array}{rr}19 . & 2.60 \\
12 . & 9.59 \\
1 . & 12.59 \\
22 . & 12.59 \\
27 . & 1.60 \\
14 . & 2.60 \\
9 . & 3.60 \\
8 . & 7.60\end{array}$ & & $\begin{array}{r}-89 \\
-157 \\
-178 \\
-115 \\
-179 \\
-238 \\
-90 \\
-272\end{array}$ & $\begin{array}{l}2,4 \\
3,3 \\
2,9 \\
3,9 \\
2,5 \\
2,4 \\
8,1 \\
3,3\end{array}$ & $\begin{array}{l}3,9 \\
7,0 \\
7,1 \\
6,5 \\
6,2 \\
9,0\end{array}$ \\
\hline $\begin{array}{l}\text { Ne } 44 \\
\text { Ne } 9 \\
\text { Ne } 45 \\
\text { Ne } 10 \\
\text { Ne } 46 \\
\text { Ne } 51 \\
\text { Ne } 52\end{array}$ & $\begin{array}{l}\text { Oberhausen } \\
\text { Oberhausen } \\
\text { Oberhausen } \\
\text { Oberhausen } \\
\text { Oberhausen } \\
\text { Oberhausen } \\
\text { Oberhausen }\end{array}$ & & 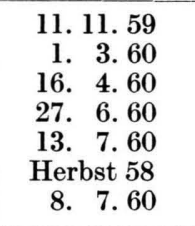 & $\begin{array}{l}0,149 \\
0,270 \\
0,162 \\
0,271 \\
0,146 \\
0,176 \\
0,136\end{array}$ & $\begin{array}{l}-269 \\
-347 \\
-197 \\
-244 \\
-253 \\
-248 \\
-168\end{array}$ & $\begin{array}{l}2,5 \\
1,8 \\
3,2 \\
2,0 \\
2,8 \\
2,0 \\
3,7\end{array}$ & $\begin{array}{l}4,7 \\
6,1 \\
6,5 \\
6,9 \\
5,1 \\
4,3 \\
6,3\end{array}$ \\
\hline $\begin{array}{ll}\mathrm{Ne} & 2 \\
\mathrm{Ne} & 3 \\
\mathrm{Ne} & 4 \\
\mathrm{Ne} & 5 \\
\mathrm{Ne} & 6 \\
\mathrm{Ne} & 7 \\
\mathrm{Ne} & 8\end{array}$ & $\begin{array}{l}\text { Lohhof } \\
\text { Lohhof } \\
\text { Lohhof } \\
\text { Lohhof } \\
\text { Lohhof } \\
\text { Lohhof } \\
\text { Lohhof }\end{array}$ & $\begin{array}{r}9^{00} \\
1700 \\
8^{00} \\
17^{00} \\
9^{00} \\
6^{00} \\
9^{00}\end{array}$ & $\begin{array}{rr}28 . & 2.62 \\
28 . & 2.62 \\
1 . & 3.62 \\
1 . & 3.62 \\
2 . & 3.62 \\
2 . & 3.62 \\
3 . & 3.62\end{array}$ & $\begin{array}{l}0,111 \\
0,111 \\
0,134 \\
0,107 \\
0,117 \\
0,093 \\
0,122\end{array}$ & $\begin{array}{l}-137 \\
-\quad 98 \\
-123 \\
-\quad 42 \\
-132 \\
-\quad 16 \\
-\quad 64\end{array}$ & $\begin{array}{l}16,8 \\
14,2 \\
14,0 \\
15,5 \\
11,2 \\
16,5 \\
13,6\end{array}$ & $\begin{array}{l}23,4 \\
19,7 \\
23,5 \\
20,8 \\
16,4 \\
19,1 \\
20,7 \\
\mathbf{2 0 , 5}\end{array}$ \\
\hline $\begin{array}{l}\text { Ne } 12 \\
\text { Ne } 13 \\
\text { Ne } 14 \\
\text { Ne } 15 \\
\text { Ne } 16 \\
\text { Ne } 17 \\
\text { Ne } 18 \\
\text { Ne } 19 \\
\text { Ne } 20\end{array}$ & $\begin{array}{l}\text { Lohhof } \\
\text { Lohhof } \\
\text { Lohhof } \\
\text { Lohhof } \\
\text { Lohhof } \\
\text { Lohhof } \\
\text { Lohhof } \\
\text { Lohhof } \\
\text { Lohhof }\end{array}$ & & $10 .-17.4 .62$ & $\begin{array}{l}0,077 \\
0,075 \\
0,077 \\
0,079 \\
0,080 \\
0,075 \\
0,081 \\
0,083 \\
0,071\end{array}$ & $\begin{array}{r}4 \\
44 \\
22 \\
26 \\
-\quad 12 \\
4 \\
30 \\
39 \\
4\end{array}$ & $\begin{array}{l}17,7 \\
19,8 \\
18,6 \\
15,4 \\
15,9 \\
18,0 \\
15,3 \\
18,0 \\
18,8\end{array}$ & $\begin{array}{l}17,7 \\
19,8 \\
18,6 \\
15,4 \\
15,9 \\
18,0 \\
15,5 \\
18,6 \\
18,8 \\
17,6\end{array}$ \\
\hline $\begin{array}{l}\text { Ne } 22 \\
\text { Ne } 23 \\
\text { Ne } 25 \\
\text { Ne } 26 \\
\text { Ne } 27 \\
\text { Ne } 28 \\
\text { Ne } 29 \\
\text { Ne } 30\end{array}$ & $\begin{array}{l}\text { Lohhof } \\
\text { Lohhof } \\
\text { Lohhof } \\
\text { Lohhof } \\
\text { Lohhof } \\
\text { Lohhof } \\
\text { Lohhof } \\
\text { Lohhof }\end{array}$ & $\begin{array}{r}13^{30} \\
17^{20} \\
7^{30} \\
10^{30} \\
13^{45} \\
17^{00} \\
7^{50} \\
11^{45}\end{array}$ & $\begin{array}{ll}\text { 3. } & 7.62 \\
\text { 3. } & 7.62 \\
\text { 4. } & 7.62 \\
\text { 4. } & 7.62 \\
\text { 4. } & 7.62 \\
\text { 4. } & 7.62 \\
\text { 5. } & 7.62 \\
5 . & 7.62\end{array}$ & $\begin{array}{l}0,103 \\
0,093 \\
0,088 \\
0,085 \\
0,088 \\
0,080 \\
0,076\end{array}$ & $\begin{array}{r}10 \\
38 \\
14 \\
39 \\
65 \\
67 \\
-\quad 13 \\
-\quad 2\end{array}$ & $\begin{array}{l}17,3 \\
17,5 \\
18,9 \\
21,2 \\
21,7 \\
23,0 \\
\\
19,3\end{array}$ & $\begin{array}{l}22,3 \\
20,3 \\
20,8 \\
22,4 \\
23,9 \\
23,0 \\
\\
21,2 \\
\overline{22,0}\end{array}$ \\
\hline $\begin{array}{l}\text { Ne } 32 \\
\text { Ne 33 } \\
\text { Ne 34 } \\
\text { Ne 35 } \\
\text { Ne 36 } \\
\text { Ne 37 } \\
\text { Ne } 38\end{array}$ & $\begin{array}{l}\text { Lohhof } \\
\text { Lohhof } \\
\text { Lohhof } \\
\text { Lohhof } \\
\text { Lohhof } \\
\text { Lohhof } \\
\text { Lohhof }\end{array}$ & $\begin{array}{r}11^{30} \\
14^{00} \\
16^{30} \\
19^{00} \\
22^{00} \\
7^{30} \\
10^{00}\end{array}$ & $\begin{array}{l}29.10 .62 \\
29.10 .62 \\
29.10 .62 \\
29.10 .62 \\
29.10 .62 \\
30.10 .62 \\
30.10 .62\end{array}$ & $\begin{array}{l}0,090 \\
0,089 \\
0,082 \\
0,131 \\
\\
0,109 \\
0,092\end{array}$ & $\begin{array}{r}34 \\
31 \\
42 \\
-\quad 64 \\
-\quad 71 \\
-\quad 82 \\
-\quad 48\end{array}$ & $\begin{array}{l}21,6 \\
22,2 \\
21,7 \\
12,4 \\
10,3 \\
13,5 \\
14,7\end{array}$ & $\begin{array}{l}24,2 \\
24,6 \\
22,4 \\
20,4 \\
19,6 \\
18,4 \\
16,9 \\
\mathbf{2 1 , 0}\end{array}$ \\
\hline $\begin{array}{l}\mathrm{Ne} 39 \\
\mathrm{Ne} 40 \\
\mathrm{Ne} 41 \\
\mathrm{Ne} 42 \\
\mathrm{Ne} 43\end{array}$ & $\begin{array}{l}\text { Lohhof } \\
\text { Lohhof } \\
\text { Lohhof } \\
\text { Lohhof } \\
\text { Lohhof }\end{array}$ & & $\begin{array}{l}15.12 .62 \\
15.12 .62 \\
15.12 .62 \\
15.12 .62 \\
15.12 .62\end{array}$ & $\begin{array}{l}0,071 \\
0,075 \\
0,075 \\
0,078 \\
0,083\end{array}$ & $\begin{array}{l}62 \\
48 \\
49 \\
31 \\
41\end{array}$ & $\begin{array}{l}23,1 \\
23,9 \\
21,5 \\
19,6\end{array}$ & $\begin{array}{l}23,1 \\
23,9 \\
21,5 \\
19,6 \\
\overline{22,0}\end{array}$ \\
\hline
\end{tabular}

Tab. 1. 


\begin{tabular}{|c|c|c|c|c|c|c|c|}
\hline Nr. & Entnahmeort & Uhrzeit & $\begin{array}{c}\text { Entnahme- } \\
\text { datum }\end{array}$ & $\begin{array}{c}\mathrm{H}_{2} / \mathrm{He}- \\
\text { Verhältnis }\end{array}$ & $\begin{array}{l}\text { D-Gehalt } * * \\
\text { in } \% / 00\end{array}$ & $\begin{array}{c}\text { T-Gehalt } \\
\text { in } 10^{5} \mathrm{~T} . \mathrm{U} .\end{array}$ & $\begin{array}{c}\text { korrigierter } \\
\text { T-Gehalt } \\
\text { in } 10^{5} \mathrm{~T} . \mathrm{U} .\end{array}$ \\
\hline $\begin{array}{l}\mathrm{Ne} 47 \\
\mathrm{Ne} 48\end{array}$ & $\begin{array}{l}\text { Lohhof } \\
\text { Lohhof }\end{array}$ & & $\begin{array}{l}\text { 2. } 63 \text { ? } \\
2.63 \text { ? }\end{array}$ & $\begin{array}{l}0,071 \\
0,090\end{array}$ & $\begin{array}{l}-69 \\
-63\end{array}$ & $\begin{array}{l}18,2 \\
17,1\end{array}$ & $\begin{array}{l}18,2 \\
19,5\end{array}$ \\
\hline $\begin{array}{l}\mathrm{Ne} 49 \\
\mathrm{Ne} 50\end{array}$ & $\begin{array}{l}\text { Lohhof } \\
\text { Lohhof }\end{array}$ & & $\begin{array}{r}10.8 .63 \\
9.8 .63\end{array}$ & $\begin{array}{l}0,086 \\
0,091\end{array}$ & $\begin{array}{l}17 \\
24\end{array}$ & $\begin{array}{l}22,3 \\
21,5\end{array}$ & $\begin{array}{l}24,0 \\
24,3 \\
\overline{\mathbf{2 4 , 2}}\end{array}$ \\
\hline $\begin{array}{l}\text { Ne } 53 \\
\text { Ne } 54 \\
\text { Ne } 55 \\
\text { Ne } 56 \\
\text { Ne } 57 \\
\text { Ne } 58\end{array}$ & $\begin{array}{l}\text { Lohhof } \\
\text { Lohhof } \\
\text { Lohhof } \\
\text { Lohhof } \\
\text { Lohhof } \\
\text { Lohhof }\end{array}$ & $\begin{array}{l}10^{00} \\
17^{00} \\
16^{00} \\
17^{00} \\
12^{000}\end{array}$ & $\begin{array}{r}30.10 .63 \\
31.10 .63 \\
3.11 .63 \\
5.11 .63 \\
7.11 .63 \\
\text { 8. } 11.63\end{array}$ & $\begin{array}{l}0,121 \\
0,181 \\
0,099 \\
0,106 \\
0,103 \\
0,106\end{array}$ & $\begin{array}{r}-182 \\
-312 \\
-15 \\
-106 \\
24 \\
-\quad 12\end{array}$ & $\begin{array}{l}15,7 \\
10,8 \\
20,8 \\
14,8 \\
21,4 \\
22,1\end{array}$ & $\begin{array}{l}25,2 \\
24,2 \\
23,6 \\
19,5 \\
27,6 \\
29,3 \\
\overline{\mathbf{2 4 , 9}}\end{array}$ \\
\hline $\begin{array}{l}\mathrm{Ne} 59 \\
\mathrm{Ne} 60\end{array}$ & $\begin{array}{l}\text { Oberhausen } \\
\text { Lohhof }\end{array}$ & $12^{00}$ & $\begin{array}{c}\text { Dezember } 63 \\
29 . \quad 1.64\end{array}$ & $\begin{array}{l}0,765 \\
0,140\end{array}$ & $\begin{array}{l}-540 \\
-164\end{array}$ & $\begin{array}{r}2,5 \\
10,0\end{array}$ & $\begin{array}{l}24,3 \\
17,5\end{array}$ \\
\hline $\begin{array}{l}\mathrm{Ne} 61 \\
\mathrm{Ne} 62 \\
\mathrm{Ne} 63\end{array}$ & $\begin{array}{l}\text { Lohhof } \\
\text { Lohhof } \\
\text { Lohhof }\end{array}$ & $\begin{array}{r}11^{00} \\
1700 \\
8^{00}\end{array}$ & $\begin{array}{ll}20 . & 2.64 \\
20 . & 2.64 \\
21 . & 2.64\end{array}$ & $\begin{array}{l}0,113 \\
0,096\end{array}$ & $\begin{array}{l}-113 \\
-234 \\
-56\end{array}$ & $\begin{array}{l}16,1 \\
11,3 \\
18,0\end{array}$ & $\begin{array}{l}22,7 \\
18,7 \\
21,6 \\
\mathbf{2 1 , 0}\end{array}$ \\
\hline $\begin{array}{l}\text { Ne } 64 \\
\text { Ne } 65 \\
\text { Ne } 66 \\
\text { Ne } 67 \\
\text { Ne } 68 \\
\text { Ne } 69 \\
\text { Ne } 70 \\
\text { Ne 71 } \\
\text { Ne 72 } \\
\text { Ne } 73 \\
\text { Ne } 74 \\
\text { Ne } 75 \\
\text { Ne } 76 \\
\text { Ne } 77 \\
\text { Ne } 78 \\
\text { Ne } 79 \\
\text { Ne } 80 \\
\text { Ne } 81 \\
\text { Ne } 82 \\
\text { Ne } 83 \\
\text { Ne } 84 \\
\text { Ne } 85\end{array}$ & $\begin{array}{l}\text { Hamburg } \\
\text { Lohhof } \\
\text { Lohhof } \\
\text { Lohhof } \\
\text { Lohhof } \\
\text { Raum Hamburg } \\
\text { Lohhof } \\
\text { Lohhof } \\
\text { Wedel/Hamburg } \\
\text { Lohhof } \\
\text { Lohhof } \\
\text { Lohhof } \\
\text { Lohhof } \\
\text { Lohhof } \\
\text { Lohhof } \\
\text { Lohhof } \\
\text { Lohhof } \\
\text { Lohhof } \\
\text { Lohhof } \\
\text { Lohhof } \\
\text { Lohhof } \\
\text { Lohhof }\end{array}$ & $\begin{array}{l}12^{00} \\
11^{00} \\
12^{00} \\
11^{00}\end{array}$ & $\begin{array}{c}25.3 .64 \\
11.4 .64 \\
1 .-10.4 .64 \\
\text { Januar } 64 \\
\text { 15.1.-20.2.64 } \\
\text { Ende April } 64 \\
\text { Ende April } 64 \\
15.5 .64 \\
\text { Ende Mai } 64 \\
3.6 .64 \\
14 . \quad 6.64 \\
5 .-25.9 .64 \\
28.9 .64 \\
22.11 .64 \\
6.12 .64 \\
22.12 .64 \\
5.2 .65 \\
21.3 .65 \\
25.3 .65 \\
29.4465 \\
10.5565 \\
28.6 .65\end{array}$ & $\begin{array}{l}0,105 \\
\\
0,094 \\
0,113 \\
0,087 \\
0,091 \\
\\
0,083 \\
0,077 \\
0,091 \\
0,088 \\
0,101 \\
0,102 \\
0,101 \\
0,099 \\
0,228 \\
0,097 \\
0,148 \\
0,097 \\
0,096 \\
0,107 \\
0,097\end{array}$ & $\begin{array}{r}-53 \\
-147 \\
-138 \\
-194 \\
-\quad 59 \\
-\quad 97 \\
-\quad 22 \\
6 \\
-\quad 51 \\
16 \\
-149 \\
-\quad 68 \\
-\quad 9 \\
33 \\
-399 \\
-18 \\
-338 \\
18 \\
15 \\
-136 \\
-73\end{array}$ & $\begin{array}{l}36,8 \\
11,9 \\
20,5 \\
12,0 \\
18,8 \\
30,7 \\
18,0 \\
22,2 \\
22,4 \\
18,6 \\
20,6 \\
15,9 \\
16,9 \\
29,9 \\
29,2 \\
10,6 \\
26,3 \\
14,3 \\
30,4 \\
73,3 \\
18,3 \\
22,1\end{array}$ & $\begin{array}{l}46,0 \\
18,2 \\
24,0 \\
17,0 \\
20,5 \\
35,0 \\
23,0 \\
23,0 \\
22,4 \\
21,2 \\
22,6 \\
20,0 \\
21,4 \\
37,2 \\
36,2 \\
30,1 \\
31,8 \\
26,2 \\
36,5 \\
88,5 \\
24,5 \\
26,8\end{array}$ \\
\hline
\end{tabular}

* Die mit L bezeichneten Proben lagen als Wasser vor. Ihr T-Gehalt wurde von G. IsrakL gemessen mit einem Fehler von $\pm 15 \%$. Alle weiteren sind „Rohneonproben “, deren Fehler im allgemeinen $\pm 5 \%$ beträgt.

** \#ezogen auf SMOW, vgl. Gl. (1).

Tab. 1. Luftwasserstoffproben. Mittelwerte in Fettdruck.

in zwei Gruppen mit verschiedenen Korrelationsgeraden. Für die Proben aus der zweiten Hälfte von 1962 finden wir einen Extrapolationswert von etwa $-250 \%$, der mit dem von Begemann und Friedman gefundenen Wert übereinstimmt ${ }^{7,8}$. Dieser Wert wurde von den genannten Autoren auf die Beimischung von Wasserstoff zurückgeführt, der über die Wassergas-Reaktion produziert wird. Für die

8 B. Gonsior, I. Friedmann u. D. Ehhalt, J. Geophys. Res. 68, 3753 [1963].
1963 entnommenen Proben ergibt die Extrapolation jedoch $\delta=-600 \%$. Dieser Wert weist auf $\mathrm{Zu}$ mischung von elektrolytisch hergestelltem Wasserstoff hin, dessen D-Gehalt um $-700 \%$ o beträgt ${ }^{4,12}$. Es ist daraus zu schließen, daß in Lohhof Wasserstoff aus beiden Herstellungsverfahren zugemischt wird. Als Quelle ist wohl eine benachbarte Reinigungsanlage für Wasserstoff anzusehen.

Man muß also damit rechnen, daß die interessierenden Isotopengehalte des atmosphärischen Wasser- 
stoffs verfälscht sind durch lokal beigemischten Wasserstoff industrieller Herkunft. Da die industrielle Wasserstoffproduktion von natürlichem Wasser ausgeht, dessen spezifische T-Aktivität wenigstens tausendmal kleiner ist als die von atmosphärischem Wasserstoff, verdünnt der lokal beigemischte Wasserstoff nur den T-Gehalt. Der ursprüngliche Gehalt kann rekonstruiert werden, wenn man den Anteil des industriellen Wasserstoffs in der Probe kennt. Tatsächlich läßt sich dieser Anteil auf zwei verschiedene Weisen bestimmen, nämlich einmal aus dem $\mathrm{H}_{2} / \mathrm{He}$ Konzentrationsverhältnis in der Rohneonprobe, zum anderen aus der D/T-Korrelation.

Man geht davon aus, daß - abgesehen von der beschriebenen lokalen Beimischung industriellen Wasserstoffs - die Gehalte der Atmosphäre an Wasserstoff, Helium und Neon genügend konstant sind; ferner, daß bei der Luftverflüssigung die Fraktionierung der nichtkondensierbaren Gase unter normalen Betriebsbedingungen so gering ist, $\mathrm{da} ß$ das ursprüngliche $\mathrm{H}_{2} / \mathrm{He} / \mathrm{Ne}$-Verhältnis in der angesaugten Luft praktisch erhalten bleibt. Gerechtfertigt scheint diese Annahme deshalb, weil bei normaler Fahrweise der Verflüssigungsanlage das in der angesaugten Luft enthaltene Neon praktisch quantitativ in der Rohneonfraktion aufgefangen wird. Helium sollte wegen seiner weitaus geringeren Löslichkeit in flüssigem Stickstoff dann ebenfalls quantitativ gewonnen werden. Demnach wird man die gefundene Erhöhung der $\mathrm{H}_{2} / \mathrm{He}$-Verhältnisse zum weitaus größeren Teil der Beimischung von industriellem Wasserstoff zuschreiben.

Eine weitere Bestätigung für die gemachten Annahmen ergibt sich aus der Korrelation zwischen T-Aktivität und dem $\mathrm{H}_{2} / \mathrm{He}$-Konzentrationsverhältnis (vgl. Abb. 2). In Abb. 2 ist der T-Gehalt der Proben gegen das $\mathrm{H}_{2} / \mathrm{He}-\mathrm{Verhältnis}$ aufgetragen. Es sind hier wieder die in Lohhof während der zweiten Hälfte 1962 und während 1963 entnommenen Proben aufgetragen, da während dieser Zeit der mittlere T-Gehalt des Wasserstoffs einigermaßen konstant war. Die Korrelation ist recht eindeutig: Die höchsten T-Konzentrationen sind stets mit dem natürlichen $\mathrm{H}_{2} / \mathrm{He}$-Verhältnis verknüpft - wenn die Luft also keine industrielle Wasserstoff-Beimischung enthält. Andererseits sind die $\mathrm{H}_{2} / \mathrm{He}-$ Verhältnisse zum

9 Diesen Wert erhält man durch Mittelung über die korrigierten T-Gehalte der in der zweiten Hälfte 1962 und 1963 entnommenen Proben.

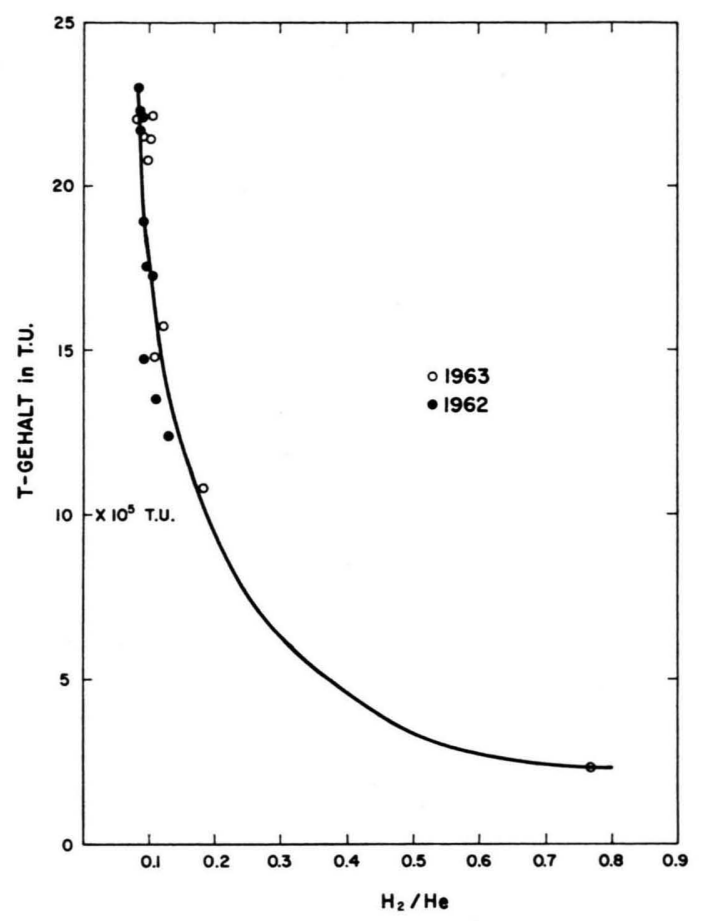

Abb. 2. Korrelation zwischen T-Gehalt und $\mathrm{H}_{2} / \mathrm{He}-$ Verhältnis für die gleichen Proben wie in Abb. 1. Das natürliche $\mathrm{H}_{2} / \mathrm{He}$ Verhältnis von 0,08 ist durch die vertikale Linie angedeutet, höhere Werte wurden durch die Beimischung von industriellem Wasserstoff verursacht (vgl. Text).

Teil beträchtlich erhöht und die T-Gehalte dann entsprechend niedrig. Die Messungen werden durch die eingezeichnete Hyperbel recht gut beschrieben. Die Hyperbel ist aus folgenden Daten berechnet: Ungestörtes $\mathrm{H}_{2} / \mathrm{He}$-Verhältnis $=0,08 ;$ mittlere ungestörte T-Konzentration in Lohhof $=23 \cdot 10^{5} \mathrm{TU}^{9}$. Diese Korrelation stützt die oben gemachten Annahmen, nämlich einmal, daß der beigemischte Wasserstoff sehr wenig Tritium enthält, zum anderen, daß die Fraktionierung der nicht kondensierbaren Gase bei der Luftverflüssigung tatsächlich gering ist. Das hier angenommene $\mathrm{H}_{2} / \mathrm{He}$-Verhältnis von 0,08 , das dem Maximum der Häufigkeitsverteilung der in Tab. 1 aufgeführten Proben entspricht, führt bei einer He-Konzentration von 5,24 ppm (GLÜCKAUF ${ }^{10}$ ) zu einer Wasserstoffkonzentration von $0,42 \mathrm{ppm}$. Dieser Wert liegt innerhalb des Schwankungsbereichs der bisher gemessenen, natürlichen Konzentrationen ${ }^{11}$.

10 E. Guueckauf, Composition of atmospheric air, in: Compendium of Meteorology, S. 3 (T. F. Malone ed.), American Meteorological Society, Boston 1952.

11 E. Glueckauf u. G. P. Kitt, Quart. J. Roy. Meteorol. Soc. 83, 522 [1957]. 
Eine ähnliche Kurve erhält man für den D-Gehalt 4, 12. Aus der Extrapolation auf das natürliche $\mathrm{H}_{2} / \mathrm{He}$-Verhältnis erhält man den D-Gehalt des „natürlichen“ Wasserstoffs zu $\delta=+80 \%$.

Aus dem bisher gesagten ergeben sich zwei Möglichkeiten, die Verfälschung des wahren T-Gehaltes durch die industrielle Beimischung von $\mathrm{H}_{2}$ zu korrigieren:

1. Da der beigemischte Wasserstoff praktisch kein Tritium enthält, ist der gemessene T-Gehalt einfach zu multiplizieren mit dem Quotienten aus gemessenem $\mathrm{H}_{2} / \mathrm{He}$-Verhältnis dividiert durch das natürliche $\mathrm{H}_{2} / \mathrm{He}$-Verhältnis von 0,08 . Der Fehler dieses Korrekturfaktors sollte kleiner als $10 \%$ sein.

2. Die zweite Möglichkeit geht von $\operatorname{der} \mathrm{T}-\mathrm{D}$ Korrelation aus, wobei angenommen wird, daß der natürliche D-Gehalt von $80 \%$ ouch für andere Entnahmeorte und Zeiten gilt. Hier wird der Extrapolationswert $\mathrm{T}=0, \delta=-250 \%_{0}$ (bzw. $-600 \%_{0}$ ) mit dem Punkt des gemessenen D. und T. Gehaltes verbunden und die Gerade bis zum natürlichen D. Gehalt extrapoliert. Der zugehörige T-Wert liefert die ungestörte T-Konzentration. Wegen der zweifachen Extrapolation, ferner wegen der Möglichkeit, $\mathrm{da} ß$ beide Arten, nämlich elektrolytisch und über die Wassergas-Reaktion produzierter Wasserstoff in variablem Verhältnis zugemischt werden und der D-Gehalt des beigemischten Wasserstoffs deshalb nicht mehr konstant ist, könnte diese Methode zu groben Ungenauigkeiten führen. Im Rahmen unserer Messungen zeigt sich jedoch eine befriedigende Übereinstimmung zwischen beiden Methoden, sofern eine eindeutige T - D-Korrelation vorliegt.

Die korrigierten T-Werte sind ebenfalls in Tab. 1 aufgenommen. Normalerweise wurde mit Hilfe des $\mathrm{H}_{2} / \mathrm{He}$-Verhältnisses korrigiert. Nur wenn keine $\mathrm{H}_{2} / \mathrm{He}$-Messungen vorlagen, wurde die zweite Korrekturmethode verwendet. Die Korrekturfaktoren liegen im allgemeinen in dem Bereich von 1,0-2,0.

\section{Diskussion}

Die in Tab. 1 aufgeführten korrigierten T-Werte ergeben den in Abb. 3 gezeigten Anstieg im T-Gehalt

12 D. EhHalt, vorgetragen bei dem CACR Symposium on Atmospheric Chemistry, Circulation and Aerosols, Visby, Sweden, 18-25 August 1965; erscheint in Tellus.

13 Hier wurde ein Extrapolationswert $\delta=250 \%$ und ein maximaler D-Gehalt von $+180 \%$ beobachtet und für die Korrektur (nach Methode 2) verwendet. des atmosphärischen Wasserstoffs. Da für 1961 keine eigenen Werte vorliegen, sind hier Messungen von Gonsion $^{12 a}$ eingefügt ${ }^{13}$.

Der vorliegende Verlauf des T-Anstiegs in Wasserstoff weicht gänzlich von dem Anstieg ab, der für die anderen bombenerzeugten Radionuklide, also Kohlenstoff-14, Tritium in Regen und die Spaltprodukte beobachtet wurde.

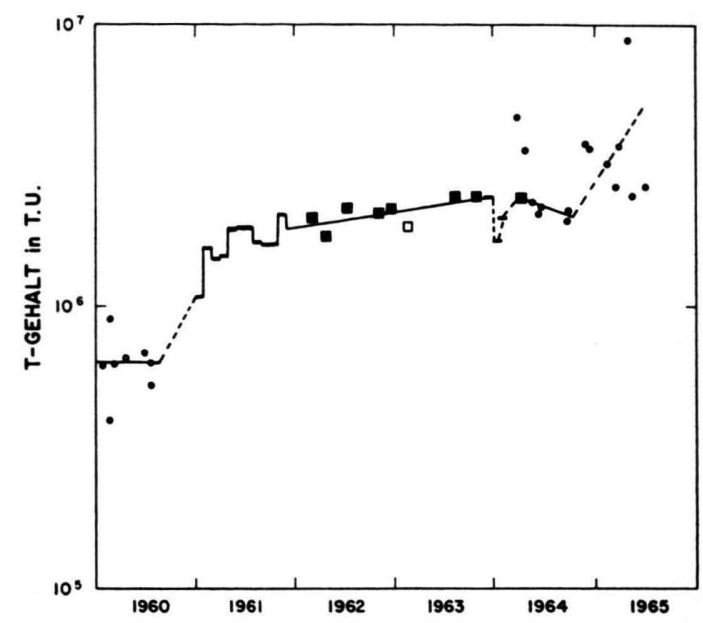

Abb. 3. Der mittlere Tritium-Anstieg von 1960 bis 1965. Die Proben wurden meistens in Lohhof (bei München) entnommen (vgl. Tab. 1). Die T-Messungen während 1961 (an Proben aus Ludwigshafen sind der Arbeit von Gonsior ${ }^{12}$ a entnommen. Die Striche bedeuten Mittelwerte über einen Monat, die Rechtecke Mittelwerte über mehrere Proben oder kürzere Zeiten. Die Kreise deuten Einzelmessungen an.

Diese Nuklide weisen während 1962 und, verstärkt noch, während 1963 als Folge der 1961 und 1962 wiederaufgenommenen thermonuklearen Tests einen beträchtlichen Aktivitätsanstieg auf. Vergleicht man damit die T-Aktivität des atmosphärischen Wasserstoffs, so zeigt sich ebenfalls ein starker Anstieg, der jedoch vor Januar 1961 einsetzt, also lange vor Beginn der ersten russischen Testserie, und der sich 1962 und 1963 immer mehr abflacht. Ein wesentlicher Beitrag der Tests 1961 und 1962 zu dem gefundenen Aktivitätsanstieg scheint damit ausgeschlossen.

Nun beobachtet man für 1961 eine deutliche Variation, die sich als ein „Frühjahrsmaximum“ 14

12a B. Gonsior, I. Friedmann u. G. Lindenmayr, Proc. 6th Intern. Conf. Radiocarbon and Tritium Dating, Pullmann Wash., June 7-11, 1965, USAEC CONF-650 652, p. 549.

14 C. E. Junge, Air Chemistry and Radioactivity, Academic Press, New York and London 1963. 
deuten läßt. Dies würde darauf hinweisen, daß tatsächlich tritierter Wasserstoff von der Stratosphäre aus zugemischt wird. Dann liegt es nahe, die Erklärung für das besondere Verhalten der troposphärischen T-Konzentration in Wasserstoff darin zu suchen, daß der beobachtete Anstieg auf solches Tritium zurückzuführen ist, das während der Testserien 1958 in die hohe Stratosphäre injiziert wurde und erst nach einer Verzögerung von $2-3$ Jahren in der Troposphäre erscheint. Ein solches Verhalten ist für die Isotope Rh-102 und Cd-109, die während atmosphärischer Tracerversuche in große Höhen injiziert wurden, tatsächlich beobachtet worden ${ }^{15}$. Die vorgeschlagene Deutung des T-Anstiegs im atmosphärischen Wasserstoff ist in einer separaten Arbeit ausführlich diskutiert ${ }^{12}$. Wie dort gezeigt wird, sind bereits der Castle-Test sowie die Tests im Spätjahr 1955 und Redwig im Frühjahr 1956 von einem um etwa 2 Jahre verzögerten Anstieg im T-Gehalt des Wasserstoffs gefolgt worden. Demzufolge wäre zu erwarten, daß der im Herbst 1961 und 1962 injizierte tritierte Wasserstoff während 1964 in der Troposphäre erscheint. Tatsächlich scheinen einzelne Proben ( $\mathrm{Ne} \mathrm{64,} \mathrm{Ne} 69)$ im Frühjahr bereits eine erste Zumischung anzudeuten, der eigentliche Anstieg scheint sich aber erst nach Oktober 1964 abzuzeichnen.

\section{Ergebnis}

Die vorgeschlagene Erklärung für die Herkunft des troposphärischen, tritierten Wasserstoffs besagt, $\mathrm{da} \beta$ dieser von Atombombentests herrührt, also stratosphärischen Ursprungs ist, daß seine Zufuhr

15 M. I. Kalkstein, J. Geophys. Res. 68, 3835 [1963]. zur Troposphäre jedoch - im Gegensatz zu den übrigen bombenerzeugten Radionukliden - erst nach einer Verzögerungszeit von etwa zwei Jahren einsetzt, danach aber jahreszeitlich in der üblichen Weise variiert. Mit dieser Hypothese läßt sich unter Zuhilfenahme der zugänglichen Information über die Testserien ein troposphärischer Anstieg berechnen und an die Meßpunkte anpassen, wobei als Parameter die stratosphärische und troposphärische Aufenthaltsdauer von tritiertem Wasserstoff eingehen. Die bisherigen Messungen reichen jedoch nicht aus, diese Aufenthaltsdauern quantitativ festzulegen. Vorläufig läßt sich nur sagen, daß die stratosphärische Aufenthaltsdauer, die man abschätzt, so kurz ist, daß eine Vernichtung des Wasserstoffs durch chemische Reaktion nahegelegt wird ${ }^{12}$.

Eine wesentliche genauere Aussage sollte sich durch Weiterverfolgen des gegenwärtigen Anstiegs (und späteren Abfalls) der Tritium-Aktivität atmosphärischen Wasserstoffs gewinnen lassen. Als Probenprogramm scheinen uns dazu wöchentlich . integrierte Proben angemessen - möglichst unter Einschluß einiger Proben von der Südhalbkugel. Falls die hier entwickelten Vorstellungen richtig sind, sollte sich dann die Frage nach der atmosphärischen Aufenthaltsdauer von tritiertem Wasserstoff beantworten und daraus weiter ein Hinweis auf die atmosphärische Aufenthaltsdauer des Wasserstoffs selbst finden lassen.

Herrn Prof. Dr. O. Haxel und Dr. K. O. Münnich danken wir für ihr förderndes Interesse und ihre Unterstützung. Herr Dipl.-Phys. K. KNotr und besonders Herr cand. phys. U. Zimmermann waren uns bei der Probenvorbereitung und massenspektrometrischen Analyse behilflich. Die Untersuchung wurde von der Deutschen Forschungsgemeinschaft durch Sachmittel gefördert. 